\title{
Open-Ended Measurement of Whole-Body Movement: A Feasibility Study
}

\author{
Michael T. M. Finn ${ }^{\mathrm{a}, \mathrm{b}, \bigotimes}$, Connor L. Smith $^{\mathrm{a}} \&$ Michael R. Nash ${ }^{\mathrm{a}}$ \\ ${ }^{a}$ Group for the Empirical Study of Psychodynamic Processes and Psychotherapy, The University of Tennessee, Knoxville \\ ${ }^{\mathrm{b}}$ Department of Psychiatry, SUNY-Upstate Medical University, Syracuse, New York
}

\begin{abstract}
As the importance of embodiment emerges for psychology, there is a need to advance methodology for measuring the dynamics of movement in an open-ended fashion. Such a tool should be versatile across contexts and track spontaneous and natural movement with minimal constraints. We test the feasibility of a method for measuring whole-body movement over time that attempts to meet this need. We use a motion capture system comprised of two Microsoft Kinect version 2.0 cameras and iPiSoft Motion Capture software, and compare its estimates of magnitude rotational velocity and whole-body movement complexity (multivariate multiscale sample entropy; MMSE) to that of a gold standard motion capture system across a variety of movement sequences. The candidate system satisfactorily estimated the instantaneous velocity of 13 body segments in agreement with the gold standard system across movement sequences demonstrating initial feasibility of this process. Summary calculations of velocity by sequence and MMSE calculations were also in high agreement with the gold standard, crucially suggesting that the candidate system could pick up on the complex dynamics of movement over time. The candidate system was feasible and demonstrates preliminary validity for general use in the tracking of continuous human movement for clinical and experimental psychology. We also provide R code and sample data for the importing and processing of movement data exported from iPiSoft Motion Capture Studio.
\end{abstract}

Keywords $\backsim$ motion capture, human movement, complexity, behavior measurement. Tools $\backsim$ iPi Motion Capture, R.

Acting Editor $\square$ Denis Cousineau (Université d'Ottawa)

Reviewers

- One anonymous reviewer

\section{- finnm@upstate.edu}

MTMF: 0000-0003-2490-2562; CLS: 0000-0002-3535-551X; MRN: n/a

10.20982/tqmp.14.1.p038

\section{Introduction}

Progress in the theoretical approaches to embodiment and embodied cognition has informed much about how one's own movement is related to psychological phenomena such as decision-making, semantic knowledge, and perception (Lakoff \& Johnson, 1999; Gallagher, 2006). Empirical research has demonstrated the relevance of continuous, dynamic movement in directing interpersonal psychological processes, through interactional synchrony, for example (e.g., Paxton \& Dale, 2013; Tschacher, Ramseyer, \& Koole, 2017), and the relevance of these processes in psychotherapy process (Ramseyer \& Tschacher, 2011) and psychopathology ((e.g., Galbusera, Finn, \& Fuchs, 2016).

Phenomenology emphasizes the role of action in struc- turing aspects of experience and the self (Sheets-Johnstone, 2011; Gallagher \& Zahavi, 2012). Informing these advances, the phenomenologist Merleau-Ponty offered the metaphor of human behavior as not necessarily a static "thing" and neither as an "idea" (2006, p. 127), but as a temporally-extended process - a kind of "kinetic melody gifted with meaning” (Merleau-Ponty, 2006, p. 130). Like a melody, behavior can be understood as an integrated, autocorrelated, and potentially complex process. Gathering dynamic, continuous data grounded in physical terms would allow the empirical psychologist to examine major questions related to embodiment and intersubjectivity: how does the structure of a person's movement reflect aspects of their self-experience? How might aspects of interpersonal relation emerge as a system of moving persons? In 
order to assess these questions as naturally-occurring processes, a measure is needed that is open to the possibilities of human movement, capable of indexing movement dynamically, and flexible to different tasks.

There is considerable literature on biomechanical measurement and kinesiology, which covers a whole range of questions about the moving human body. Naturally, there are extensive and highly advanced instrumentation for the collection, processing, and analysis of kinetic (e.g., energy and force) and kinematic (e.g., velocity and acceleration) variables (Winter, 2009; Robertson, Caldwell, Hamill, Kamen, \& Whittlesey, 2013). The physics of human movement can be complex, the instrumentation advanced, and the data processing extensive. For these reasons, if there is to be any sustainable study of movement for the questions of psychology, there must be a manageable and open-ended approach that draws out the relevant features of biomechanics and describes a process with some confidence in its validity.

\section{Meeting Methodological Needs}

One historic convergence of psychology and biomechanics in tracking continuous movement lies in the study of human gait patterns (see Jaspers, 1963, for an early synthesis). In the case of gait, there are various measures from biomechanics, like inter-stride intervals, which capture aspects of the movement (e.g., Mentiplay et al., 2015). Likewise, there are specific uses of biomechanical measurement of behavior, like coordination of hand movement (e.g., Liddy et al., 2017). These approaches provide initial confidence and validity of a more open-ended approach, where similar instrumentation could be applied to situations where there are minimal constraints on movement and naturally occurring, systemic dynamics of the whole body are more central. As such, a method that can describe movement of the whole body and its parts in a way that can be used across forms of movement may be an ideal addition to the methodological toolbox in psychology.

Rotational velocity is one such index that can be used to meet these needs. Occurring in three-dimensional space, rotational velocity can characterize the movement of body segments as they rotate at joints together and navigate space. Rotational velocity is typically described as the change in degrees or radians per second of Euler angles, which capture each of the three dimensions of rotation (Robertson et al., 2013, p. 50). It can be transformed from a three-dimensional matrix into a one-dimensional scalar rather easily by calculating the Euclidean norm of the three rotational dimensions. Not only does this make the interpretation and computation of movement data easier, but it would also harmonize well with neuroscientific methodology, like EEG and fMRI, which can also be processed into sets of scalar values over time. The feasibility of this index would also inform the use of a similar index of movement: kinetic energy

Dynamic structure of movement. Studies in biomechanics have applied algorithms that calculate the variability of movement over time in terms of complexity, or meaningful structural richness (Newell, Deutsch, Sosnoff, \& MayerKress, 2006). These approaches offer powerful tools for characterizing complexity in natural systems, and shifting attention toward the structural characteristics of variability has led to progress in understanding dynamic structure of healthy, optimal performance across human systems. A line of research in this effort has demonstrated that optimal heart rate styles are richly complex, while unhealthy styles have been shown to either be highly predictable (e.g., highly regular beats) or highly random (e.g., atrial fibrillation; Costa, Goldberger, \& Peng, 2002). Similar models have been applied to discover increases in the dynamic complexity of brain activity among individuals with schizophrenia after taking antipsychotics (Takahashi et al., 2010), to discriminate between fMRI data of young and elderly adults (Sokunbi, 2014), and to characterize the difference between healthy and unhealthy human gait and balance dynamics (Costa, Peng, Goldberger, \& Hausdorff, 2003). While this represents a potential measure for characterizing whole-body movement dynamics across contexts, it needs to be explored with the chosen measure of rotational velocity under open-ended conditions.

Instrumentation. Passive motion capture systems are standard in the measurement of movement in biomechanics. While they are extremely accurate, they require significant preparation of a single participant with markers and in the modeling of their morphology 'from the bottom up.' These markers allow a multi-camera system to passively record location in three-dimensional space at a high accuracy. Figure 1a shows a participant with markers attached to his body. These were used only by the passive motion capture system and are irrelevant to the candidate active system introduced below. This process also requires expertise on the part of the experimenter and a large, dedicated space for the sophisticated network of cameras involved. If motion capture is to be smoothly integrated into psychology research, finding more feasible and more inconspicuous instrumentation is paramount.

An active motion capture system, exemplified by the Microsoft Kinect hardware, is a promising response to this concern. These systems are active in the sense that they actively fit a human figure to depth data 'from the top down.' By way of active modeling, these systems introduce an additional layer of estimation into the collection of raw movement data, naturally introducing additional error. Increased error in the measurement of movement dynamics

The Quantitative Methods for Psychology 
Figure 1 \& Corresponding Frame of Video, Visualization of Data Tracked from the Active Motion Capture System Rotated to a Reverse Perspective and Video Sample (Available Online). These images are sourced solely from the active motion capture system. The notations of " 1 " and "2" in part b label the cameras in global space. The image in part a was captured from the camera 1 video. Part c is a video that can be streamed or downloaded: tiny.utk.edu/Figure1c.

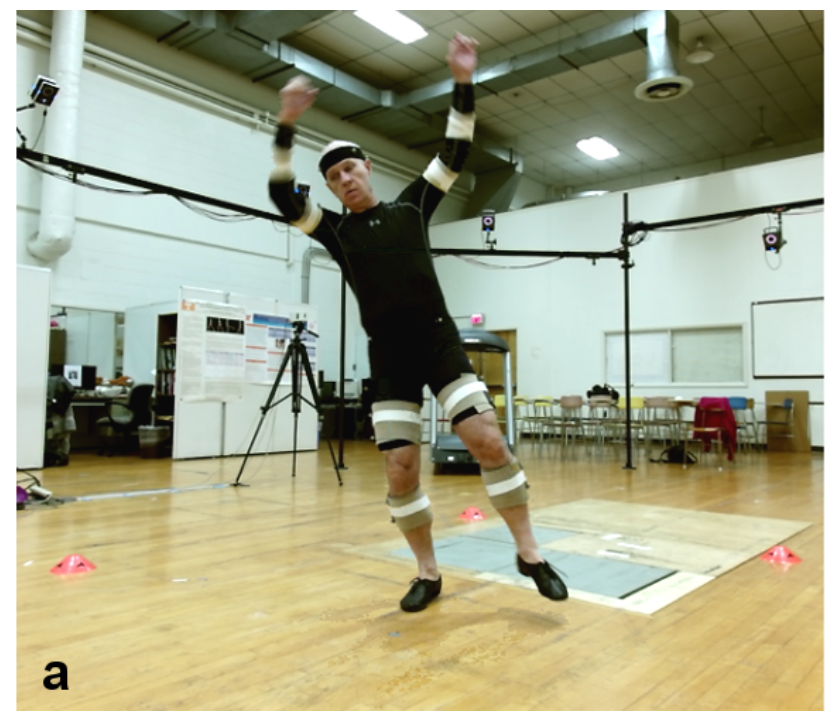

by active systems may be tolerable given accessibility advantages, but only if they can be thoroughly validated. Active systems seem well positioned for use in movement research that requires the collection of three-dimensional dynamic data. Where large numbers of participants are frequent, a markerless, active system provides a way to measure the movement of people more immediately, without applying materials to a participant or having them wear any specialized equipment. A frame of tracked movement corresponding to that of the still image from the video in Figure 1a is given in Figure 1b. Also see Figure 1c online at tiny.utk.edu/Figure1c for a video visualization of the movement data across the same sequence.

While the Kinect may not reach the accuracy needed for fine-grained biomechanical questions achievable by passive systems, the accuracy and rate of measurement sufficiently estimate spatiotemporal characteristics of movement. While there are several studies on the accuracy of the Kinect version 2.0 data (e.g., Clark et al., 2015; Geerse, Coolen, \& Roerdink, 2015; Liddy et al., 2017; Mentiplay et al., 2015), it remains to be established whether this system can record a general measure like magnitude rotational velocity in an open-ended fashion.

Thus, a study was designed to examine the agreement between the estimates of this active system with a passive system on magnitude rotational velocity in body segments. This study also presents the development of a general pro-

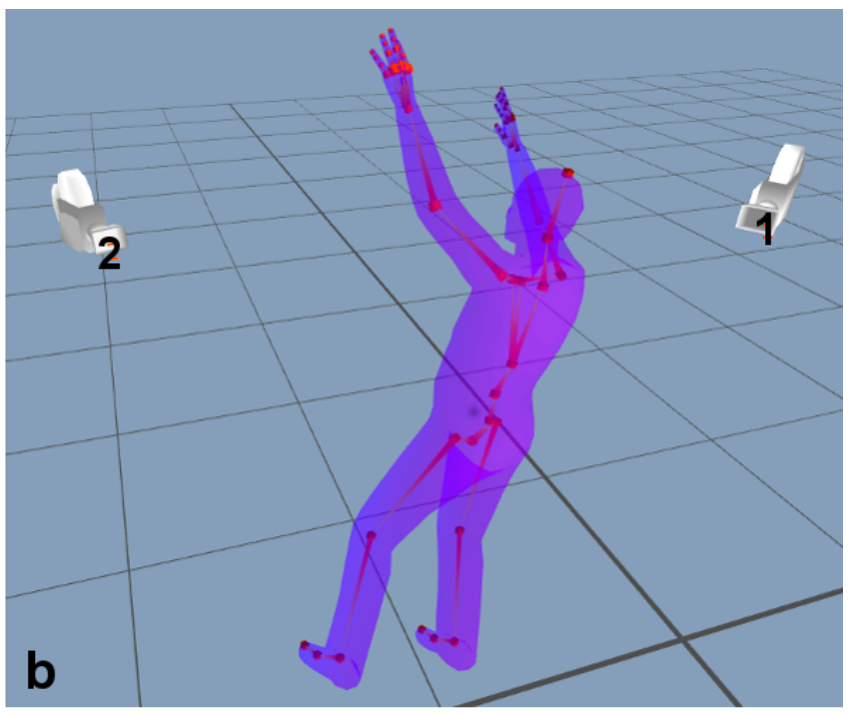

cedure for processing movement data and for analyzing the dynamic structure of movement.

\section{Method}

\section{Participants}

Three individuals completed a number of movement sequences for this study. Two were actors studying for their Masters of Fine Arts (MFA) in Acting (one male, one female) who performed a series of seven varied movement sequences. These two actors had previous coursework in movement and corporeal mime. The other participant was a professor of theater who specialized in movement training. He performed six sequences of corporeal mime from the tradition of Etienne Decroux (Decroux, 1985; Leabhart, 1989). The institution's Internal Review Board approved all study procedures and all individuals gave written informed consent for participation in the study and for scientific presentation or publication of videos or images of their participation. With such involved and rich data collection, a small sample of individuals with a series of sequences performed each was deemed sufficient to test the feasibility of the method at hand.

\section{Experimental Procedure}

After the calibration of instrumentation and basic preparation of participants for both movement systems, they 
Table 1 - Movement Sequences Performed

\begin{tabular}{|c|c|c|c|c|}
\hline \multirow[b]{2}{*}{ Sequence } & \multirow[b]{2}{*}{ Duration Limit } & \multicolumn{3}{|c|}{ Participant } \\
\hline & & Female MFA Actor & Male MFA Actor & Professor of Theater \\
\hline Free treadmill & $1 \mathrm{~min}$ & $\mathrm{x}$ & $\mathrm{x}$ & \\
\hline Paced treadmill & $1 \mathrm{~min}$ & $\mathrm{x}$ & $\mathrm{x}$ & \\
\hline Random & $1 \mathrm{~min}$ & $\mathrm{x}$ & $\mathrm{x}$ & \\
\hline Swaying & $1 \mathrm{~min}$ & $\mathrm{x}$ & $\mathrm{x}$ & \\
\hline Mock stress interview & $5 \mathrm{~min}$ & $\mathrm{x}$ & $\mathrm{x}$ & \\
\hline Ground walking & None & $\mathrm{x}$ & $\mathrm{x}$ & \\
\hline The Rope & None & $\mathrm{x}$ & $\mathrm{x}$ & $\mathrm{x}$ \\
\hline $\begin{array}{l}\text { Flick of the Seahorse's Tail } \\
\text { Actions of Agriculture }\end{array}$ & None & & & $\mathrm{x}$ \\
\hline Scythe & None & & & $\mathrm{x}$ \\
\hline Pulling a cart & None & & & $\mathrm{x}$ \\
\hline Pitchfork & None & & & $\mathrm{x}$ \\
\hline Sowing seeds & None & & & $\mathrm{x}$ \\
\hline
\end{tabular}

were asked to perform a series of movement sequences. The two MFA participants each received identical instructions for movement sequences and performed them in the same order: treadmill walking freely and governed by a metronome, one minute sequences of random and swaying movement, the simulation of an interview procedure, a corporeal mime piece, and a brief walk through the capture area on the ground. These tasks were selected to provide a varied database of samples with widely different properties intended to push the limits of the active system's movement tracking capacities. Refer to Table 1 for a summary of these movement sequences.

\section{Movement Sequences for the MFA Students}

Treadmill walking: free and paced movement. Participants were asked to walk on a commercial-grade treadmill both freely and with their steps paced by a metronome for one minute each. Speed was held constant within the two trails of the participants, but was set to a participantdetermined comfortable speed. The metronome, which gave the constant indication of beats per minute (bpm) by a "click" sound, was set at $120 \mathrm{bpm}$.

Random and recurring sequences. For further samples of movement with varying properties, the MFA actors were instructed to conduct two further movement sequences: random, to move around randomly for one minute and swaying, to sway in place for another minute. These sequences allowed for the exploration of validity in the contexts of more periodic movement and more open-ended movement.

Mock interview. As one planned application of movement measurement to psychology was through a welldefined stress induction paradigm, the Trier Social Stress Test (Kirschbaum, Pirke, \& Hellhammer, 1993), MFA actors were asked to undergo the administration of the test for a 5-minute duration in character. In summary, they were instructed to interview as a character of their choosing for a “dream job”. The inclusion of this in our experimental procedure allowed for an even more open-ended movement sequence, tracking natural speaking behavioral.

Corporeal mime: The Rope. MFA actors were asked to perform a prepared corporeal mime piece. In this sequence, the participant moves as if they hand off a rope. Each participant performed the sequence three times during their recording.

Ground walking. To assess the active system's ability to capture kinematics at a short duration, the MFA actors were instructed to walk from the back of the capture area toward and through the space between the two Kinect cameras.

\section{Movement Sequences for the Professor of Theater}

The male professor of theater performed The Rope and five additional corporeal mime sequences, including a series of four sequences from the Etienne Decroux corporeal mime tradition called the Actions of Agriculture. This is a series of four movement sequences that portray features of agricultural work: sowing seeds, pulling a cart, cutting crops with a scythe, and using a pitchfork. The professor of theater's fifth movement sequence was called Flick of the Seahorse's Tail, in which he portrayed the dynamics of a seahorse tail through bilateral arm movements. In terms of testing the motion capture instrumentation, these corporeal mime sequences provided rich and varied samples of human movement. 


\section{Instrumentation}

The gold standard: a passive motion capture system. A 9-camera MX3/T10 $1000 \mathrm{~Hz}$ motion analysis system, a passive motion capture system, was used as the gold standard of movement measurement in this study. This is a highly accurate state-of-the-art motion capture system used as a standard for biomechanical research (Windolf, Götzen, \& Morlock, 2008). Providing a three-dimensional perspective on movement in the capture area, this allows for highly accurate and reliable recording of kinematics.

Participants were outfitted with specialized light reflectors by experts in the operation of this system (see Figure 1a for an image of a participant wearing markers). These are designed to be minimally obtrusive, so as not to distort the participant's natural movement. These reflectors are also specially designed to interface with the multiple cameras to produce three-dimensional location data. Before performing the movement sequences, participants stood in the center of the recording space for a baseline snapshot, which this system used as a template for modeling all future movement of this participant.

The candidate: an active motion capture system. In 2013, Microsoft released a second version of their Kinect camera system, which involves a hardware camera and a linked software development kit (SDK) allowing for data collection and analysis with a personal computer running Windows. Like its predecessor, this version measures human movement in three dimensions over time through a markerless method. That is, a method that does not require the placement of sensors or reflective markers on the participant's body. This system records depth data using infrared sensors. Such data contain three-dimensional information, which can be actively modeled to a human form.

Some approaches to collecting these data use custom programming to extract position data of the built-in skeletal; figure provided by the SDK (e.g., Clark et al., 2012). Third-party software also exists (e.g., Brekelmans, 2016) that captures this raw data directly from the Kinect recording. By these systems, motion capture data of the body segments are directly written and usually only from the perspective of one camera. Though the data can be filtered or cleaned for outliers, the core movement modeling is essentially unalterable for these systems. Video might be recorded incidentally, but once recorded, the movement data are written and cannot be remodeled or resampled after the fact.

The software system utilized in this study is made up of iPi Recorder 3.2.5.47 (iPi Soft LLC, 2016b) and the iPi Motion Capture Studio (Basic) 3.4.16.212 with the Biomechanics Add-on (iPi Soft LLC, 2016a). iPi Recorder is free to download and use from, but iPi Motion Capture Stu- dio is required for the modeling of movement and is neither open source nor open-access software. This software takes a unique approach to active motion capture with the Kinect camera (and is compatible with other cameras like the PlayStation Eye). Rather than directly recording skeletal movement data, iPi Recorder first records the depth data over time. In a second step, this video is "tracked" in iPi Motion Capture Studio. It is only at this stage that movement data are modeled.

For the purposes of an open-ended approach to the measurement of movement, this step between depth data recording and movement tracking offers significant advantages. One can supervise the movement tracking process and multiple cameras synthesize into a more accurate picture of depth. Regarding the supervision of tracking, one can observe the software estimating movement relative to the observable video. Though infrequent, an active motion capture system has the potential of misidentifying or 'losing track' of a body segment through the course of recording, compounding into massive tracking errors. In an active motion capture system that directly records movement data, it is difficult to notice this loss of the segment tracking since there is no visual comparison from which to supervise, and no backup of the depth recording from which to remodel. Being able to visually compare and potentially revise the model in reference to the actual video turns out to be a huge methodological advantage toward preserving data.

Calibration of cameras. When conducting a multicamera setup, the spatial relationship between the two cameras needs to be identified by the software before the two sets of depth data can be synthesized. See Figure 1b for a representation of the two cameras in global space. This was accomplished by recording a calibration video before any movement sequences can be recorded. An experimenter traversed the capture area with a bright light source in hand, which the iPi Motion Capture Studio software tracks across both cameras. There need not be a strict arrangement of the cameras relative to each other - all successful calibrations are equivalently functional. A second calibration video was recorded at the end of the experimental session in case the camera arrangement of the presession calibration was somehow altered (e.g., accidentally bumping a camera), but ultimately went unused in this study.

Arrangement of cameras and laptops. Two Kinect version 2.0 cameras were stationed at the edge of the passive system's pre-existing recording capture area. Since the exact arrangement of cameras is flexible, they were placed approximately 3 meters apart and at different heights. The exact values were $2.85 \mathrm{~m}$ apart and facing inward, forming a $78^{\circ}$ angle. The camera stage right was at a $.8 \mathrm{~m}$ height 
over the ground with a slight, $4.26^{\circ}$ upward tilt, with the camera stage left at a $.9 \mathrm{~m}$ height over the ground and a $0.48^{\circ}$ upward tilt. While these are exact reports on the camera positions, once the cameras are calibrated to one another in this setup, the exact positions make little difference.

As the software requires, the two cameras were linked to their own dedicated laptop. These two laptops were connected by Ethernet cable for reliable simultaneity and data transfer of the two recordings. One laptop-camera pairing served as the master controller of the recording process, dictating the start and stop times of the of the slave laptop-camera pairing. Cameras were situated on standard video camera tripods set to the sides of a table that had the laptops on it. iPi Recorder software made the depth data recordings and facilitated the networking of the machines.

Recording procedure. For the active motion capture system, participants are required to begin each movement sequence in a T-Pose (i.e., standing upright with arms held outward, parallel with the ground). This allows the software to easily initiate the modeling of the participant's body. Participants were instructed to hold the T-Pose for around 2-3 seconds to gather more than enough data for the identification of the body contours after which the participant began the designated movement sequence. The passive motion capture system recording began immediately following the end of the T-Pose stance.

Tracking and refining procedure with iPi Motion Capture Studio. The movement sequences were paired with a calibration file when imported into iPi Motion Capture Studio. A model of each participant was visually fitted to the videos for tracking. This is a quick process requiring no more than 30 seconds. Parameters like height, arm length, and leg length, were adjusted to fit the still frame of the participant standing in a T-Pose. Videos were then tracked forward in time. Whenever a gross error of tracking occurred relative to the original video, the tracking was paused, the human skeletal model manually corrected at the erroneous moment, and the software self-corrected tracking going forward. This was rare in our experience, adding anywhere from one to five minutes of additional processing time beyond the software's tracking. After forward tracking was completed, each video was backward refined. Backward refinement essentially refits the participant's frame-by-frame pose going backwards in time, from the end to the beginning, providing subtle improvements to the initial tracking.

\section{Common Measurement Across Both Systems}

Body segments and groupings. Movement of body segments were measured in both systems as Head, Chest, and Hips, with left and right measurements of Upper Arms,
Forearms, Thighs, Shins, and Feet. For all sequences, these body segments were also grouped by summing their respective values for each frame into Upper Body (Head, Chest, Upper Arms, and Forearms) and Lower Body (Hip, Thighs, Shins, Feet). Finally, a Total segments group was created by summing all body segments on their respective values for each frame. Thus, there were 16 values created in total for each movement sequence with 13 body segments and 3 calculated segment groupings.

Frame of reference. The kinematic data from both systems were calculated relative to the global frame of reference. Each body segment was measured relative to the ground and described by kinematics independent of their position relative to other body segments. This can be contrasted with a frame of reference where the kinematics of each body segment is treated relative to their parent joint, for example, the Upper Arm's movement relative to the Chest. Since both systems are calibrated to the global frame of reference, it provided a uniform and identical frame between the two motion capture systems for rotational data.

Sampling rate. We proceeded with $30 \mathrm{hz}$ as the standard sampling rate for all measures. This was the fastest sampling rate permitted for the active motion capture system. The passive motion capture system was sampled at $100 \mathrm{hz}$, which was transformed into $30 \mathrm{hz}$ by upsampling by a magnitude of 3 to $300 \mathrm{hz}$ and downsampling by a magnitude of 10.

Aligning data. Data were aligned to the correct frame by first gathering an estimate of the corresponding frames between visualizations of the movement data. Second, crosscorrelation analyses were performed in $\mathrm{R}$ to discover the exact active and passive system frame with maximum correspondence and prepare the data accordingly for agreement analyses.

\section{Data Processing}

Magnitude rotational velocity. Magnitude rotational velocity captures the movement of each body segment. In this project, it was measured as degrees per second of rotation relative to the global reference system (relative to the room). It was the primary metric under evaluation in this study as common across the active and passive systems, with the values of the passive system treated as a fixed gold standard. It is also the metric from which dynamic structure calculations are calculated for further evaluation of agreement. The degree to which the active system measures magnitude velocity accurately directly informs the reliability of future calculations of kinetic measures, such as kinetic energy.

Filtering data. It is common practice to filter biomechanical data to better model true kinematic values, typically 
with a low-pass Butterworth filter (Winter, 2009, p. 35). Passive system data of the markers were collected at 100 $\mathrm{hz}$, and a residual analysis determined that an optimal filter was a low-pass Butterworth filter at $10 \mathrm{hz}$.

For the active system, filtering was optimized relative to the processed passive system data as the first step in the data analysis process. The results of this analysis informed the filter order parameters and cut-off frequency for all following agreement analyses. Filtering was performed in $\mathrm{R}$ using the signal package (Developers, 2014) and a custom function wrapper used to assess agreement for each movement sequence at many filter combinations. This process was intended to maximize the overall performance of the active motion capture system for this study and establish defaults for further research to work from. The values derived from this process were then incorporated into the default values of the $\mathrm{R}$ code including the supplementary materials.

$\mathbf{R}$ Code and Supplementary Files. Instructions and $\mathrm{R}$ code for importing and processing data into magnitude velocity over time and for creating the summary values of a movement sequence are provided in supplementary materials. One script loads the function, ipi_read, which can be used to directly import the tab-delimited files produced by iPi Motion Capture Studio Biomechanics Addon (exported according to the export profile also provided). The script, movement, loads a function that processes the imported database into channels of magnitude velocity and summary values, exporting them as .csv files. The movement function also has options for applying a Butterworth filter (default values being the optimal values derived in this project), changing beginning and end frames to be processed.

Complexity. Complexity was operationalized as sample entropy using two closely related algorithms, multiscale sample entropy and multivariate sample entropy. The sample entropy calculation forms the core of these algorithms, known as SampEn (Richman \& Moorman, 2000). Overall, SampEn was designed as an open-ended calculation of the degree of patterning in data over time.

The algorithm works by assessing the probability that, over a sequence of a given $m$ points, that $m+1$ falls within an acceptable tolerance, $r$, at other $m+1$ samples across the time series. Richman and Moorman summarize this algorithm as "precisely the conditional probability that two sequences within a tolerance $r$ for $m$ points remain within $r$ of each other at the next point.” ( p. H2042). T calculates the conditional probability of the number of template matches $m+1$ given the number of template matches at $\mathrm{m}$ length. To give a standard scaling, the negative natural logarithm is taken of this probability.

SampEn improves upon the approximate entropy algo- rithm (ApEn; Pincus, 1991), which was modeled to capture the mean rate of generation of information in a time series, or Kolmogorov-Sinai (KS) entropy. It is unbiased by not counting templates as matching themselves and is more robust to differences in time series length, making it a better estimate own complexity (Richman \& Moorman, 2000).

Multiscale. Multiscale sample entropy introduces a multiscale advancement in measurement to this sample entropy algorithm (MSE; Costa, Goldberger, \& Peng, 2005). Whereas its predecessors, approximate entropy and sample entropy, consider complexity across a single time scale, Costa et al. (2005) note that complex processes often happen at different time scales in real-world data. This treatment helps to preserve the entropy metric in accurately characterizing high-information variability as complex, maintaining a guiding intuition about complexity as representing "meaningful structural richness" in dynamic data (Grassberger, 1991, p. 16). The authors of MSE noticed that white noise can often erroneously register as more complex than naturally autocorrelated systems (Costa et al., 2005). By paying attention to larger time scales, MSE can observe long-range dynamic structures typical of living systems. Thus, rather than one scaled value of entropy, this approach generates a series of entropy values for an individual time series, one for each integer increase in time scale $(\varepsilon)$.

As an analogy to the changing time scales, consider a digital geographical map. One can zoom in closely to the details of a street and can, step by step, zoom out to a neighborhood, a city, a state or country, and so on. Coarsegraining a time series works this way, but by averaging across bins of time-based data. The multiscale feature of MSE does this very change in scope. For instance, $\varepsilon=10$ averages bins of every 10 frames of data, $\varepsilon=20$ across bins of every 20 frames. Thus, when recording at 30 frames per second, an MSE analysis at $\varepsilon=15$ counts patterns across half-second means.

Multivariate. The multiscale sample entropy measure has been developed further by Ahmed and Mandic to account for complexity in multiple channels of data on the same system 2011. This applies the multiscale approach while considering cross-channel pattern matching within and across subspaces of a multivariate time series. Where sample entropy requires the fixed parameters of $\mathrm{m}$, and $\mathrm{r}$, and multiscale modification, of time scale, the multivariate algorithm requires these as well as the specification of time lags among the channels of data $(\tau)$. This method generalizes sample entropy to the multivariate case at the specified $\tau$ for the specified range of time scales. This is a rigorous and computationally taxing procedure to perform, which grows more taxing as the number of channels increases and the length of the data increase. MATLAB code was publicly shared by the developers of this algorithm

The Quantitative Methods for Psychology 
and was used in this study to assess both MMSE and MSE (MSE being the 1-channel case of MMSE; Ahmed \& Mandic, 2011).

XSEDE high performance computing system. High performance computing resources were required for most exhaustive tests of MMSE (13-channels of movement data). Processing of the multiscale sample entropy algorithms was completed via an allocation of service units on the NSF-funded Extreme Science and Engineering Discovery Environment (XSEDE), which was started at the University of Illinois-Champaign (Towns et al., 2014). The resource that was utilized in this study was Comet, the highperformance computing cluster based in the San Diego Supercomputing Center (SDSC; Moore et al., 2014). For this project, Comet was accessed remotely, and was used to run the MMSE algorithm on fully prepared time series from both the active and passive systems to examine agreement on these measures.

\section{Data Analysis Strategy}

Data analyses focused on the agreement of the active system to the passive system. This is a matter of agreement between a gold standard measure and a trial measure where absolute values are relevant. Additional analyses explored trials for expected features of the multivariate multiscale sample entropy values.

Assessing agreement. Concordance correlation coefficient (rc). Statistical analyses on agreement have advanced to the calculation of concordance, which can be decomposed into accuracy and precision (Leabhart, 1989). A common measure of relatedness, Pearson correlation and its non-parametric equivalents, do not meet the needs of examining the agreement of instrumentation methods. In the case of agreement, it is usually known a priori that the systems are measuring that same object. Thus, correlation loses much relevance and the question becomes: to what extent the measures are identical (Leabhart, 1989, p. 255)? It is important to know how measures agree in terms of scaling (of which correlation is scale-independent); not only whether they are related over time or between trials, but whether they also return similar values of the phenomenon at hand.

The concordance coefficient has been developed to deal with variance between the measures in fixed or random ways, depending on the question at hand. In the case of the current study, variance was treated as fixed, considering the active system and the passive system as composing the population of interest, in comparing a trial instrument to a gold standard without intending to generalize this effect to a larger population (Lin, Hedayat, Sinha, \& Yang, 2002, p. 258-260).

While there are no certain benchmarks for the inter- pretation of concordance, it is scaled from -1 to 1 and operates similarly to the intra-class correlation coefficient, producing very similar values (Chen \& Barnhart, 2008) and might be roughly interpreted with the same rules of thumb of ICC in the social sciences (Cicchetti \& Sparrow, 1981). While much higher concordance values are considered necessary for the replacement of one method by another, this framework gives us a rough scale as to how well the active system can estimate point-values of the time series.

The concordance coefficient is used throughout the current study and is considered the primary measure of degree of agreement. The R package Agreement was used to calculate these coefficients (Yu \& Lin, 2012).

The precision component of concordance is identical to Pearson correlation, particularly a fixed effects version of the Pearson correlation in the case of assessing concordance to a fixed target (Lin et al., 2002, p. 259). Precision, then, assesses for the structural relationship between measures of the same phenomenon in a relative fashion while the accuracy component assesses for estimation of the measured value in absolute terms.

Limits of agreement. A way to capture the real-world meaning of $\mathrm{rc}$ is to present the high and low-bound $95 \%$ limits of agreement (Barnhart et al., 2016). In the current case, these would be the bounds within which one could expect the active system values to be relative to the gold standard. These values form guiding lines on BlandAltman plots, which are also generally recommended for visually assessing the agreement of two measures (Bland \& Altman, 1986). These plots show the relationship between the average of the two measures and the difference between the two measures, allowing for a depiction of any systematic errors in measurement that might occur and cannot be captured by a single coefficient like rc (e.g., more extreme errors at the high end of measurement). The $\mathrm{R}$ package BlandAltmanLeh was used to create these plots and calculate the 95\% limits of agreement (Lehnert, 2015).

\section{Three Perspectives on the Validity of the Candidate Sys- tem}

In the current study, a passive system designated as a gold standard measure for measuring movement, providing the values for an active system to estimate. Both systems concurrently measured a series of movement sequences. The agreement of active system estimates to the passive system values was assessed through three perspectives: dynamic, summary, and dynamic structure.

Dynamic. One perspective employed in this project was the dynamic agreement between the two systems, that is, comparing the continuous, frame-by-frame values of both systems for each segment and for groups of segments (in-

The Quantitative Methods for Psychology 
cluding one of all the segments summed together for each frame) within movement sequences. These agreement indices were then averaged across movement sequences for the main results. These analyses encompass large numbers of observations using core measures of agreement: the concordance coefficient ( $\mathrm{rc}$ ), precision coefficient $(\mathrm{r})$, accuracy $\left(\chi_{a}\right)$, and the $95 \%$ limits of agreement of values. The $95 \%$ lower confidence limit (CLL) of rc is also presented along with rc values.

Summary. The second perspective was the summary agreement, which involves the analysis of summaries of magnitude velocity across the whole movement sequence. That is, calculating the mean and standard deviation of movement for each segment and segment grouping across the whole sequence. This is more important to our approach than the dynamic results, since this technology will be used not to estimate single values of movement, but instead summarize sequences of movement.

Dynamic structure. The third perspective of analysis was on dynamic structure agreement. This involves analyses on sets of calculated values that reflect different elements of time series structure: autocorrelation and complexity. Autocorrelation values up to a lag of 90 frames were calculated for body segments and segment groupings for each movement sequence and compared using the suite of agreement measures. Following, agreement between systems on the measures of complexity, multiscale sample entropy and multivariate multiscale sample entropy was assessed with the same suite of analyses. Given the relatively short duration of these walking sequences, they were not long enough in duration to be included in analyses of dynamic structure agreement.

\section{Results}

Both passive and active motion capture data for all 20 movement sequences were successfully collected with no missing data. We present the agreement between the active and passive systems over time in magnitude rotational velocity in degrees per second. Being a Euclidean-normed scalar the three-dimensional velocity, magnitude velocity gives simplified direct measure of general movement for each body segment over time. That is, it reflects a general "how much movement" recorded from a body segment over time without information about which dimension of rotation (X, Y, or Z) or which direction (positive or negative). In this way, magnitude velocity could be characterized as the omnidirectional rotational speed of a body segment over time. Validating magnitude rotational velocity of each body segment in the whole-body system provides a foundation toward supporting accuracy kinetic energy calculations. The following analyses include all movement sequences of the experiment by all three participants $(n=20)$.

\section{Optimal Filtering of the Active System Data}

To proceed with the agreement analyses and evaluations of complexity measures, the optimal filtering procedure for the active motion capture data needed to be established. Maximum concordance with the passive motion captures system across movement sequences was determined as the criteria for selecting a specific low-pass Butterworth filter. Optimal filter was operationalized as the filter that, across all body segments over several movement sequences, resulted in maximum frame-by-frame concordance with corresponding passive motion capture segments.

For this analysis, we examined the 1-minute and 5minute movement sequences of the two MFA actors, 10 in total. For each movement sequence, agreement of the active to the passive system was assessed at 30 digital frequencies (.01 to .30 by .01 increments) for 6 filter orders (1-6). In addition, the agreement of unfiltered data was assessed for comparison. In total, 180 Butterworth filter settings were tested for each of the 13 body segments for 10 movement sequences. We then averaged concordance measures at each Butterworth filter across body segments to get a value of overall performance. Following, averaged concordance across movement sequences was used to derive an optimal Butterworth filter for all subsequent data analyses.

Among tested filters, the optimal low-pass Butterworth filter was a second order filter at a digital filter value of .24. See Figure 2 for a selection of five seconds of the male MFA actor's mock interview data in the calculated total magnitude velocity. The optimal filter marginally improved upon the concordance coefficient of the unfiltered data: the optimal filter had a concordance coefficient of $r_{c}=.627$ with a lower-bound 95\% confidence limit of $C L_{L}=.619$ which is larger than the unfiltered data, $r_{c}=.600, C L_{U}=.613$. The optimal filter made gains in precision to the passive motion capture system over unfiltered data with an $r=$ $.686\left(C L_{L}=.671\right)$. The unfiltered data had less precision, $r=.674\left(C L_{U}=.656\right)$, the upper-bound of which did not reach the lower-bound of the optimal filter, demonstrating a notable improvement in the optimal filter.

Accuracy decreased with the optimal filter $\left(r_{c}=.855\right.$, $\left.C L_{U}=.861\right)$ when compared to the unfiltered data $\left(r_{c}=\right.$ .870, $C L_{L}=.863$ ). Given the overall improvement in concordance and the relative importance of precision over accuracy for the purposes of dynamic analyses, we proceeded with the optimal Butterworth-filtered data as the standard for all following analyses.

The Quantitative Methods for Psychology 
Figure 2 - Five Seconds of Filtered and Unfiltered Active System Movement Data with Corresponding Passive System Data. These are data of the male MFA actor's mock interview. Units are degrees per second.

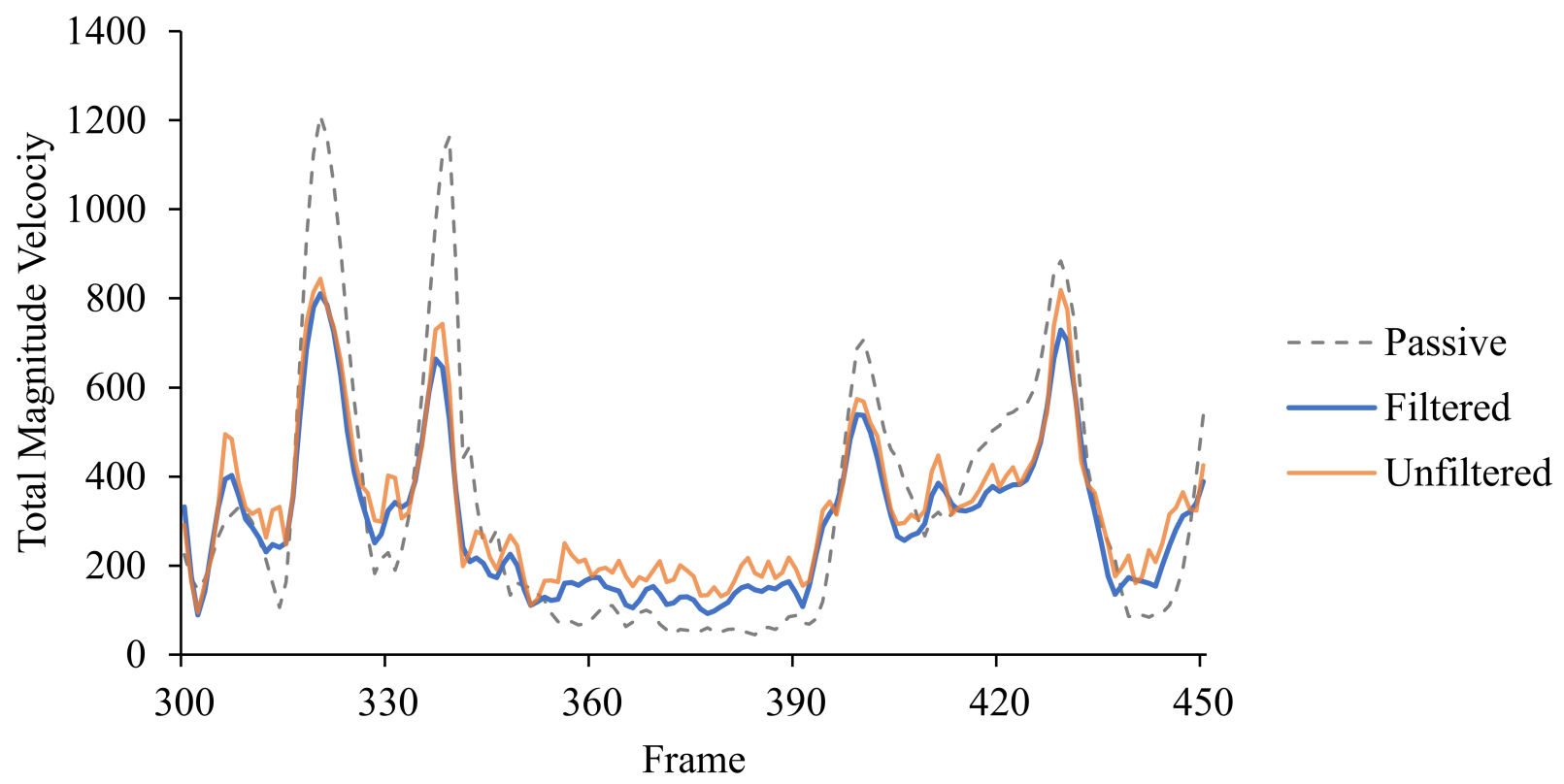

\section{Dynamic Agreement}

Agreement of the active system to the passive system over time was assessed. This focused on the ability of the active system to track the ongoing dynamics of movement. This was performed for each segment and segment grouping for each movement sequence at $30 \mathrm{hz}$. As an illustration of the data involved in a single movement sequence, see Figure 3 for the magnitude velocity of all 13 segments through the Scythe movement sequence performed by the professor of theater presented alongside these same data visualized as movement. Across body segments for all movement sequences, the active motion capture system data agreed with the passive motion capture system with an average $r_{c}=.644\left(C L_{L}=.623\right)$. This was composed of a precision of $r=.694\left(C L_{L}=.670\right)$, and an accuracy of $\chi_{a}=.883$ $\left(C L_{L}=.868\right)$. Body segments had an average rotational magnitude velocity of 57.38 degrees/sec with an average absolute error of 21.83 degrees/sec. The averaged 95\% limits of agreement ranged as follows: $-59.52 \leq-.01 \leq$ 59.33 degrees/sec. The three sequences with the largest average concordance were two of the movement sequences from the Actions of Agriculture performed by the professor of theater (Scythe and Pulling a Cart), and the male MFA actor's moving randomly sequence. The three sequences with the lowest average concordance were the male and female MFA actors' swaying sequences and the male MFA actor's The Rope sequence. It seemed that sequences involving segments with low levels of raw movement performed most poorly. For all sequences, average concordance across segments was highly significant, $p \mathrm{~s}<.01$.

The active system's continuous total values tend to underestimate the passive system's values and there are many observations that fall well outside of the $95 \%$ limits of agreement. We can roughly label an $r_{c}=.644$ as "good" for the purposes of social science research based on widely-accepted ICC benchmarks developed by Cicchetti and Sparrow (1981). For our purposes, it appears that the candidate approach was a feasible and consistent estimate of the gold standard values.

Totals. There was more agreement between the two systems' frame-by-frame summed totals of all segments, $r_{c}=$ $.676\left(C L_{L}=.661\right)$, with a much larger precision $r=.835$ $\left(C L_{L}=.819\right)$ and a somewhat lower accuracy $\left(\chi_{a}=.798\right.$, $C L_{L}=.786$ ). Average $\mathrm{p}$ values of concordance between the active and the passive systems was statistically significant, $p<.01$. With an average magnitude rotational velocity of 744.66 ( $S D=378.20$ ) for the active system, agreement on total values had the following $95 \%$ limits of agreement around mean error: $-591.63 \leq-162.98 \leq 265.68$ degrees per second. For an example of these data, see Figure 4 for the first minute of total magnitude velocity val- 
Figure 3 - A Sample of Movement Data with A Visualization of the Same Data from the Professor of Theater's Performance of the Scythe Movement Sequence. Units for magnitude velocity are degrees per second.
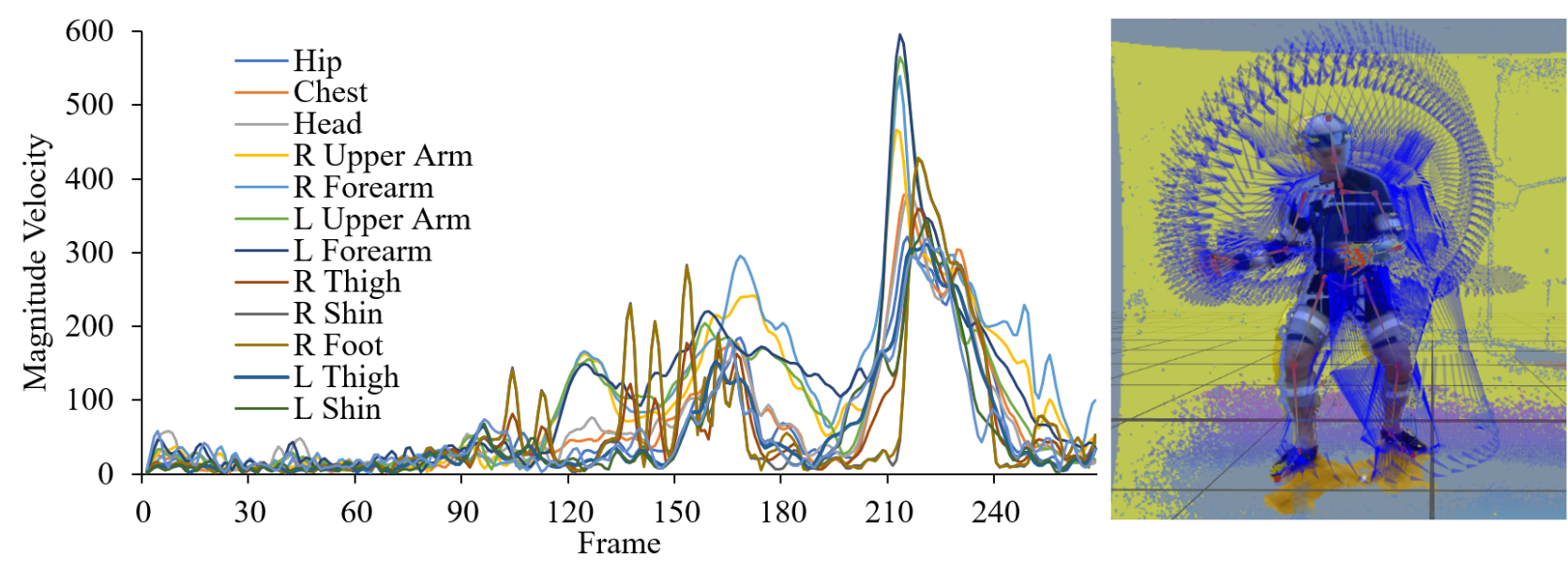

ues on the female MFA actor's mock interview. Table 2 shows the dynamic agreements of segments and segmentgroupings averaged across participants and movement sequences.

\section{Summary Agreement}

The agreement between systems on the calculated kinematic averages of body segments and segment groupings was then assessed. Average magnitude velocity was calculated for segments and groupings for each system, agreement was assessed for each movement sequence, and agreement values were averaged across movement sequences. The standard deviation of magnitude velocity was also calculated as a summary index of how much velocity values varied across the sequence and subjected to the same analysis.

Across all individual segments, the agreement between the active and passive systems on mean magnitude velocity was extremely high, $r_{c}=.956\left(C L_{L}=.939\right)$. The upper body and lower body segment groupings each had extremely high agreement with the passive system $\left(r_{c} s=\right.$ .985 and .957), while the agreement on mean total velocity was somewhat less, $r_{c}=.878$. Overall, the validity of the active system's mean magnitude velocity estimates was high. See Table 3 for a report of agreement between the two systems on segment and grouping-wise mean summary data. A Bland-Altman plot reveals a relative normality of errors with a tendency for the active motion capture system to underestimate magnitude velocity at high velocity values (Figure 5).

Agreement on standard deviations of magnitude velocity was high with an average $r_{c}=.921$ (average 95\%
$\left.C L_{L}=.890\right)$ across all individual body segments. Standard deviations of magnitude velocity for body segment groups were also in agreement between the two systems, at or above $r_{c}=.913$.

\section{Dynamic Structure Agreement and Examination: Mul- tivariate Multiscale Sample Entropy}

Multivariate multiscale sample entropy values were calculated for all movement sequences except for the MFA actors' short walking sequences. In general, we sought to examine the agreement of complexity values computed on the data produced by the active motion capture system with those produced by the gold standard. To proceed, we first needed to fix m, $\tau$, and $\mathrm{r}$ values for the MMSE algorithm. Previous research has used $m=2, \tau=1$, and $r=(.15 \times S D$ of z-scored time series) in the description of human gait (Ahmed \& Mandic, 2011), so these values were carried forward for these analyses.

Agreement was explored between the two systems on MMSE values up to $\varepsilon=20$. The systems were in more agreement for this multivariate version of the sample entropy algorithm than the univariate, $r_{c}=.768\left(C L_{L}=\right.$ .711). With an average sample entropy value of .108 $(S D=.120)$ across both systems, there was a small mean difference between the two methods (-.003) and a 95\% limits of agreement of $-.066 \leq-.003 \leq .061$. On average, concordance was significant for the dynamic structure calculations, $p<.01$.

There were two sequences with low agreement: the acting professor's Flick of the Seahorse's Tail, and the male MFA actor's swaying sequence. These are two sequences where at least a few body segments had very low variabil- 
Figure 4 - System Comparison of Total Magnitude Velocity in the First Minute of Female MFA Actor's Mock Interview. Units for magnitude velocity are degrees per second.

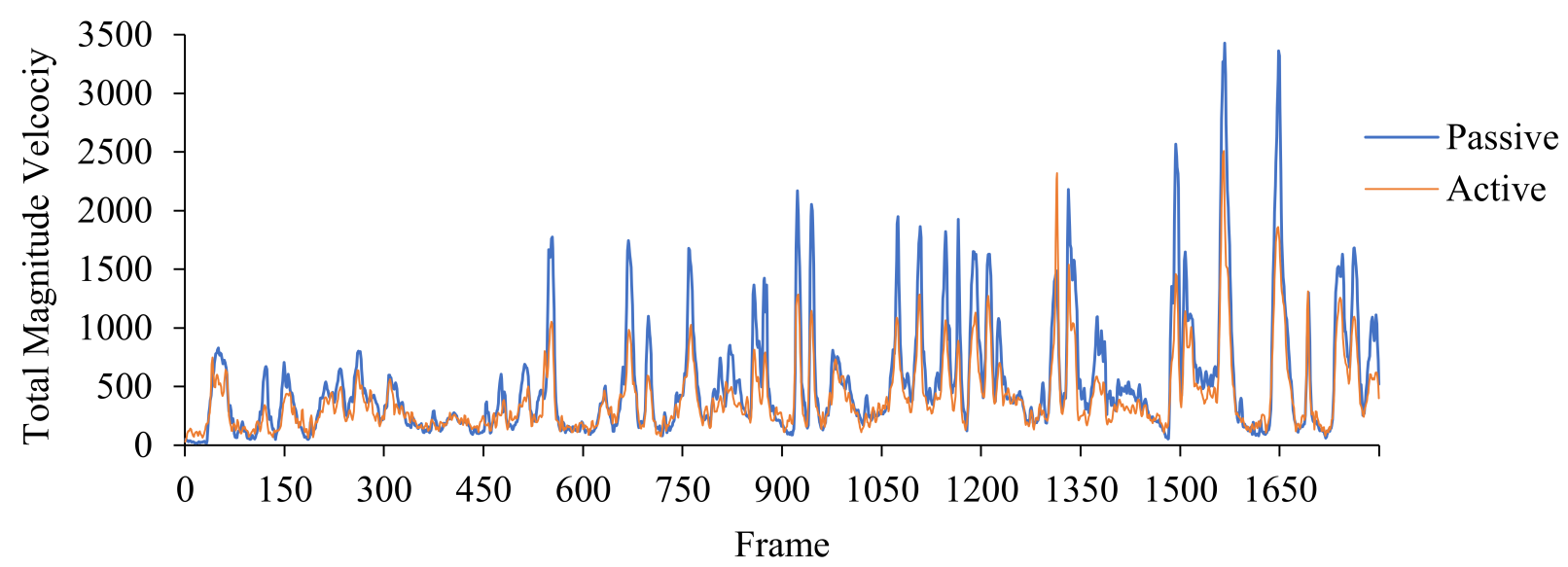

ity in movement during the sequence. For comparison, when these two removed from the calculations, average concordance increases substantially, $r_{c}=.867\left(C L_{L}=\right.$ .817).

\section{Discussion}

This project tested the validity of a way to measure movement for the purposes of basic and applied psychology research. This led to the specification of rotational magnitude velocity of body segments and segment groupings as a primary measure of interest given the possibility of this measure to index movement across contexts and in an open-ended fashion. The study also led to the development of a data collection and processing strategy that utilized an active motion capture system. The selected system had the major advantage of recording movement with the opportunity to monitor for and/or correct gross errors.

The magnitude rotational velocity provided by this system was compared in kind with gold standard instrumentation in biomechanics. Mean and standard deviation summaries of the movement sequence data were calculated and compared for both systems as well, and assessed the methods relative to the gold standard. The ability of the active system to capture aspects of dynamic structure was assessed as well, with comparisons between systems on complexity calculations.

The candidate active motion capture system could track dynamic movement - frame by frame - in moderate agreement with a gold standard measure across a variety of movement sequences. In addition, it could characterize movement across a sequence with summary measures (mean and SD of magnitude rotational velocity) in very high agreement with a gold standard measure. While the active system does not track dynamic movement in such a way that it could totally replace the gold standard motion capture system, it does seem to reliably estimate the movement dynamics of a sequence. The active system's estimate of multivariate multiscale sample entropy was in high agreement with the gold standard measure. This is a crucial finding toward the feasibility of this approach for psychology research: the candidate system could successfully track complex dynamics occurring both across channels and over time.

\section{Limitations}

There are three main limitations to the method presented in this study. First, the movement recording and data processing setup presented in this study does not record hand movement. The Microsoft Xbox Kinect system is otherwise highly developed to record hand movement, even to the specificity of the angles of joints within fingers, so it would in principle be possible to obtain hand movement if they are of interest for a given research question.

Second, this study utilized only two Kinect version 2.0 cameras in the capturing of movement. At least with a professional edition of iPi Motion Capture Studio, it is possible to record and synthesize the depth data of up to four Kinect version 2.0 cameras at once. This would require four different computers linked via a Local Area Network due to the design of the Kinect drivers, but it seems that such a setup would only improve the accuracy and reliability of the Kinect recordings. Additional cameras should only im- 
Table 2 - Dynamic agreement across all movement sequences

\begin{tabular}{|c|c|c|c|c|c|c|c|c|}
\hline \multirow[b]{2}{*}{ Segment or Grouping } & \multicolumn{4}{|c|}{$r_{c}$ Components } & \multirow[b]{2}{*}{ Mean (SD) } & \multicolumn{3}{|c|}{ 95\% Limits of Agreement } \\
\hline & $r_{c}$ & $C L_{L}$ & $\rho$ & $\chi_{a}$ & & Lower & Mean Error & Upper \\
\hline Hip & .40 & .37 & .48 & .75 & $34.61(25.42)$ & -26.29 & 7.09 & 40.47 \\
\hline Chest & .62 & .60 & .68 & .86 & $35.02(28.41)$ & -22.25 & 3.8 & 29.85 \\
\hline Head & .51 & .48 & .58 & .80 & $40.27(31.26)$ & -35.62 & 8.25 & 52.11 \\
\hline Upper Arm (R) & .70 & .68 & .72 & .93 & $62.54(48.01)$ & -57.04 & -0.93 & 55.18 \\
\hline Forearm (R) & .74 & .72 & .76 & .93 & $85.14(66.65)$ & -69.3 & 3.83 & 76.96 \\
\hline Upper Arm (L) & .67 & .65 & .69 & .94 & $62.74(47.43)$ & -57.31 & 0.58 & 58.47 \\
\hline Forearm (L) & .73 & .71 & .76 & .96 & $83.2(63.57)$ & -71.54 & 2.64 & 76.82 \\
\hline Thigh (R) & .74 & .72 & .77 & .93 & $47.69(35.14)$ & -42.47 & -1.3 & 39.88 \\
\hline Shin (R) & .72 & .71 & .78 & .90 & $59.99(47.45)$ & -62.49 & -4.66 & 53.17 \\
\hline Foot $(\mathrm{R})$ & .50 & .47 & .60 & .79 & $62.17(49.43)$ & -115.3 & -7.56 & 100.17 \\
\hline Thigh (L) & .75 & .73 & .78 & .95 & $48.42(35.4)$ & -40.22 & -0.54 & 39.13 \\
\hline Shin (L) & .78 & .76 & .82 & .94 & $60.27(49.48)$ & -57.23 & -3.11 & 51.02 \\
\hline Foot (L) & .51 & .49 & .60 & .80 & 62.59 (51.48) & -116.68 & -9.32 & 98.04 \\
\hline Averaged Across Segments & .64 & .62 & .69 & .88 & $57.28(44.55)$ & -59.52 & -0.1 & 59.33 \\
\hline Upper Body & .79 & .78 & .83 & .92 & $368.92(237.56)$ & -176.68 & 18.16 & 212.99 \\
\hline Lower Body & .67 & .65 & .77 & .84 & $375.74(201.58)$ & -252.45 & -19.4 & 213.65 \\
\hline Total & .68 & .67 & .84 & .80 & 744.66 (378.2) & -591.63 & -162.98 & 265.68 \\
\hline
\end{tabular}

Note. Segment Groupings are first summed on magnitude velocity data for each sequence, then agreement is assessed between the two systems. Upper Body is composed of Head, Chest, Upper Arms, and Forearms. Lower Body is composed of Hip, Thighs, Shins, and Feet. (L) and (R) specify left or right side of the body from the participant's perspective. Units for movement values are degrees per second. $r_{c}$ is concordance, $C L_{L}$ is lower confidence limit, $\rho$ is precision, and $\chi_{a}$ is accuracy.

prove the measurement of movement as rotational velocity.

Third, the current study did not directly address testretest reliability of the active motion capture system relative to the gold standard system. Such assessment would lend more credence to system's ability to measure movement beyond examining its feasibility and initial suggestions of validity. One caveat to this limitation is that with the approach presented in this work, the actual movement tracking process conducted by iPi Motion Capture Studio can be instantaneously compared to the raw video. That is, one can watch the system as it estimates the movement of all body segments monitor for gross errors. In the current study, it is an educated guess that correcting for gross errors led to different agreement results (e.g., correcting an arm segment tracking from being completely unhinged from the actual video). Anecdotally, when these massive losses of tracking occur, the motion capture systems rarely recover or self-correct and errors only compound over time.

\section{Directions for Dynamic Movement Processing and Analysis}

There may be ways to improve the data processing and analysis of movement. We review a few areas that emerge as potential directions for the development of the data processing and analysis of movement data. One overarching direction in this area would be to develop ways to ease the implementation of movement data collection in clinical and experimental psychology research.

Removing low-performing segments and refining segment calculations. By refining the whole-body model to remove low-performing segments, overall model reliability would increase. Of course, this should be weighed against the downside of losing a segment in the model. For example, the feet were low-performing and may not be too central to the success of whole-body modeling of movement, while the head also be relatively low-performing, but perhaps more theoretically important (see Ramseyer \& Tschacher, 2014). Similarly, somehow removing segments below a certain threshold of velocity variability may dramatically improve reliability of measurement.

Dimension reduction with segment groupings. With a set of data, even perhaps the data presented in this study, one could perform an exploratory factor analysis to determine whether there are prevailing subgroups of body segments across participants and simplify the description of whole body movement to these groups. This approach runs the risk of oversimplifying what might be a wide diversity of movement patterns across participants, but may 
Table 3 - Summary Agreement Across All Movement Sequences

\begin{tabular}{|c|c|c|c|c|c|c|c|c|}
\hline \multirow[b]{2}{*}{ Segment or Grouping } & \multirow[b]{2}{*}{$r_{c}$} & \multirow[b]{2}{*}{$C L_{L}$} & \multicolumn{2}{|c|}{$r_{c}$ Components } & \multirow[b]{2}{*}{ Mean (SD) } & \multicolumn{3}{|c|}{ 95\% Limits of Agreement } \\
\hline & & & $\rho$ & $\chi_{a}$ & & Lower & Mean Error & Upper \\
\hline Hip & .93 & .89 & .97 & .96 & $34.61(25.42)$ & -21.88 & -7.09 & 7.7 \\
\hline Chest & .98 & .96 & .99 & .99 & $35.02(28.41)$ & -15.23 & -3.8 & 7.62 \\
\hline Head & .92 & .89 & .97 & .95 & $40.27(31.26)$ & -22.28 & -8.25 & 5.78 \\
\hline Upper Arm (R) & .97 & .96 & .99 & .98 & $62.54(48.01)$ & -24.27 & 0.93 & 26.14 \\
\hline Forearm (R) & .97 & .96 & .99 & .98 & $85.14(66.65)$ & -34.55 & -3.83 & 26.9 \\
\hline Upper Arm (L) & .98 & .97 & .99 & .99 & $62.74(47.43)$ & -22.09 & -0.58 & 20.93 \\
\hline Forearm (L) & .99 & .98 & .99 & .99 & $83.2(63.57)$ & -21.56 & -2.64 & 16.29 \\
\hline Thigh (R) & .98 & .96 & .99 & .99 & $47.69(35.14)$ & -14.07 & 1.3 & 16.66 \\
\hline Shin (R) & .96 & .95 & .99 & .97 & $59.99(47.45)$ & -22.27 & 4.66 & 31.59 \\
\hline Foot (R) & .92 & .89 & .99 & .93 & $62.17(49.43)$ & -35.15 & 7.56 & 50.27 \\
\hline Thigh (L) & .98 & .97 & .99 & .99 & $48.42(35.4)$ & -13.81 & 0.54 & 14.89 \\
\hline Shin (L) & .98 & .97 & .99 & .98 & $60.27(49.48)$ & -17.51 & 3.11 & 23.72 \\
\hline Foot (L) & .90 & .88 & .99 & .91 & $62.59(51.48)$ & -36.57 & 9.32 & 55.21 \\
\hline Averages Across Segments & .96 & .94 & .99 & .97 & $57.28(44.55)$ & -23.17 & 0.1 & 23.36 \\
\hline Upper Body & .99 & .99 & .83 & .99 & $368.92(237.56)$ & -112.07 & -18.16 & 75.76 \\
\hline Lower Body & .96 & .99 & .77 & .97 & $375.74(201.58)$ & -140.08 & 19.4 & 178.9 \\
\hline Total & .89 & .99 & .84 & .90 & 744.66 (378.2) & -324.6 & 162.98 & 650.6 \\
\hline
\end{tabular}

Note. Units are degrees per second for movement values. $r_{c}$ is concordance, $C L_{L}$ is lower confidence limit, $\rho$ is precision, and $\chi_{a}$ is accuracy.

improve the parsimony of the data. Reductions along these lines would also aid in making computationally-intensive data processing, like the multiscale multivariate sample entropy calculations, possible on long duration recordings without the need to enlist supercomputing.

Kinetic and potential energy. Since we have shown preliminary validity of rotational velocity, one direction for measurement is body segment rotational kinetic energy (Robertson et al., 2013). Kinetic energy provides a scalar of movement, a one-dimensional summary of threedimensional movement.

The calculation of the rotational kinetic energy of a given body segment requires a few indices for calculation: 1) segment mass, 2) segment length, and 3) rotational velocity. Segment mass is estimated through models defining segments as proportions of the total body mass (de Leva, 1996), so its reliable measurement only depends on having the person's overall mass, while a motion capture system can be used to estimate segment length. Generally, segment length is held constant for a given participant so the results of this study suggest that the candidate system would provide all of the data necessary for these calculations.

\section{Conclusion}

The opportunity to study whole-body movement dynamics opens a methodological window into core problems at the nexus of phenomenology and psychology: self-regulation, intersubjectivity, the process of human embodiment in the world, affective consciousness, and pre-reflective and reflective experience. It allows for specifying behavior continuously, through the metaphor of kinetic melodies (Luria, 1973; Merleau-Ponty, 2006), considering the ways that neurological and other bodily systems interact - or form a coherent whole - over time.

With reliable measurement of movement dynamics, one could investigate the integration of multiple systems in a person over time. Movement data could be coupled with EEG data to explore synchrony or even causal chains between brain activity and motor behavior over time using existing statistical modeling in these areas, like dynamic causal modeling (Friston, Harrison, \& Penny, 2003). Neurophenomenology and phenomenological approaches to cognition have already empirically examined questions about temporal self-constitution to examine the process of pre-reflective awareness (Thompson, 2007; Varela, Thompson, \& Rosch, 1991). To the extent that movement dynamics are involved in the articulation of awareness, integration with EEG methodology may be a way to deeply explore perception and action.

Similarly, the convergence of movement with heart rate or skin conductance could be investigated to explore individual differences in sympathetic and parasympathetic nervous system function with movement, for example, with and without stress. These approaches might specify overregulation and underregulation dynamics of 
Figure 5 a Summary Agreement Between the Active and Passive Systems. On the left is a log-log plot of measurements by the two systems with the line of identity for reference. On the right is a Bland-Altman plot.

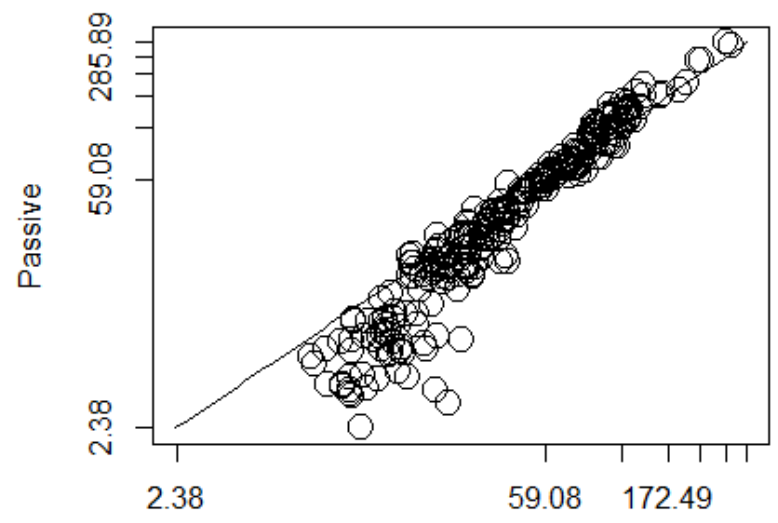

Active

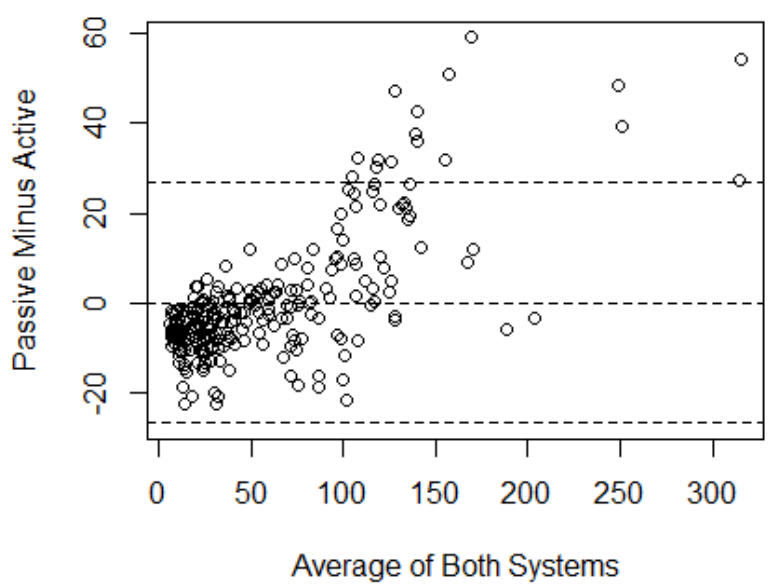

\section{References}

Ahmed, M. U. \& Mandic, D. P. (2011). Multivariate multiscale entropy: a tool for complexity analysis of multichannel data. Physical Review E, 84(6), 61918. doi:10. 1103/PhysRevE.84.061918

Barnhart, H. X., Yow, E., Crowley, A. L., Daubert, M. A., Rabineau, D., Bigelow, R., ... Douglas, P. S. (2016). Choice of agreement indices for assessing and improving measurement reproducibility in a core laboratory setting. Statistical Methods in Medical Research, 25(6), 2939-2958. doi:10.1177/0962280214534651

Bland, M. J. \& Altman, D. G. (1986). Statistical methods for assessing agreement between two methods of clinical measurement. The Lancet, 327(8476), 307-310. Retrieved from 10.1016/S0140-6736(86)90837-8

Brekelmans, J. (2016). Brekel pro body (Version 2.027). Brekel 3D. Retrieved from www.brekel.com

Chen, C.-c. \& Barnhart, H. X. (2008). Comparison of icc and ccc for assessing agreement for data without and with replications. Computational Statistics \& Data Analysis, 53(2), 554-564. doi:10.1016/j.csda.2008.09.026

Cicchetti, D. V. \& Sparrow, S. A. (1981). Developing criteria for establishing interrater reliability of specific items: applications to assessment of adaptive behavior. American Journal of Mental Deficiency, 86(2), 12737. 
Clark, R. A., Pua, Y.-h., Fortin, K., Ritchie, C., Webster, K. E., Denehy, L., \& Bryant, A. L. (2012). Validity of the microsoft kinect for assessment of postural control. Gait \& Posture, 36(3), 372-377. doi:10.1016/j.gaitpost.2012. 03.033

Clark, R. A., Pua, Y.-h., Oliveira, C. C., Bower, K. J., Thilarajah, S., McGaw, R., ... Mentiplay, B. F. (2015). Reliability and concurrent validity of the microsoft xbox one kinect for assessment of standing balance and postural control. Gait \& Posture, 42(2), 210-213. doi:10. 1016/j.gaitpost.2015.03.005

Costa, M., Goldberger, A. L., \& Peng, C.-k. (2002). Multiscale entropy analysis of complex physiologic time series. Physical Review Letters, 89(6), 68102. doi:10 . 1103 / PhysRevLett.89.068102

Costa, M., Goldberger, A. L., \& Peng, C.-k. (2005). Multiscale entropy analysis of biological signals. Physical Review E, 71(2), 21906. doi:10.1103/PhysRevE.71.021906

Costa, M., Peng, C.-K. L., Goldberger, A., \& Hausdorff, J. M. (2003). Multiscale entropy analysis of human gait dynamics. Physica A: Statistical Mechanics and Its Applications, 330(1-2), 53-60. doi:10.1016/j.physa.2003.08. 022

de Leva, P. (1996). Adjustments to zatsiorsky-seluyanov's segment inertia parameters. Journal of Biomechanics, 29(9), 1223-1230.

Decroux, E. (1985). Words on mime. In T. Leabhart (Ed.), Mime journal.

Developers, S. (2014). Signal: signal processing (Version 1.00). Retrieved from http://r-forge.r-project. org/projects/signal/

Friston, K. J., Harrison, L., \& Penny, W. (2003). Dynamic causal modelling. Neuroimage, 19(4), 12731302. doi:10.1016/S1053-8119(03)00202-7

Galbusera, L., Finn, M. T. M., \& Fuchs, T. (2016). Interactional synchrony and negative symptoms: an outcome study of body-oriented psychotherapy for schizophrenia. Psychotherapy Research, 0(0), online first, 1-13. doi:10.1080/10503307.2016.1216624

Gallagher, S. (2006). How the body shapes the mind. New York: Clarendon Press. doi:10.1093/0199271941.001. 0001

Gallagher, S. \& Zahavi, D. (2012). The phenomenological mind (2 edition). London?; New York: Routledge.

Geerse, D. J., Coolen, B. H., \& Roerdink, M. (2015). Kinematic validation of a multi-kinect v2 instrumented 10-meter walkway for quantitative gait assessments. PLoS ONE, 10(10), e0139913. doi:10 . 1371 / journal . pone.0139913

Grassberger, P. (1991). Information and complexity measures in dynamical systems. In H. Atmanspacher \& H. Scheingraber (Eds.), Information dynamics (Vol. 256, pp. 1-44). Boston, MA: Springer. doi:10.1007/978-14899-2305-9_2

iPi Soft LLC. (2016a). Ipi mocapmotion capture studio (basic) with biomechanics add-on (Version 3.4.16.212). Retrieved from http://ipisoft.com/

iPi Soft LLC. (2016b). Ipi recorder. Retrieved from http:// ipisoft.com/

Jaspers, K. (1963). General psychopathology. Manchester: Manchester University Press.

Kirschbaum, C., Pirke, K.-m., \& Hellhammer, D. H. (1993). The 'trier social stress test' - a tool for investigating psychobiological stress responses in a laboratory setting. Neuropsychobiology, 28(1-2), 76-81. doi:10.1159/ 000119004

Lakoff, G. \& Johnson, M. (1999). Philosophy in the flesh: the embodied mind and its challenge to western thought. New York: Basic books.

Leabhart, T. (1989). Modern and post-modern mime. Basingstoke: Palgrave.

Lehnert, B. (2015). BlandAltmanLeh: Plots (Slightly Extended) Bland-Altman Plots. Retrieved from https:// CRAN.R-project.org/package=BlandAltmanLeh

Liddy, J. J., Zelaznik, H. N., Huber, J. E., Rietdyk, S., Claxton, L. J., Samuel, A., \& Haddad, J. M. (2017). The efficacy of the microsoft kinecttm to assess human bimanual coordination. Behavior Research Methods, 49(3), 10301047. doi:10.3758/s13428-016-0764-7

Lin, L., Hedayat, A. S., Sinha, B., \& Yang, M. (2002). Statistical methods in assessing agreement. Journal of the American Statistical Association, 97(457), 257-270. doi:10.1198/016214502753479392

Luria, A. R. (1973). Neuropsychological studies in the ussr. A Review (Part I). Proceedings of the National Academy of Sciences of the United States of America, 70(3), 959964.

Mentiplay, B. F., Perraton, L. G., Bower, K. J., Pua, Y.-h., McGaw, R., Heywood, S., \& Clark, R. A. (2015). Gait assessment using the microsoft xbox one kinect: concurrent validity and inter-day reliability of spatiotemporal and kinematic variables. Journal of Biomechanics, 48(10), 2166-2170. doi:10.1016/j.jbiomech.2015.05. 021

Merleau-Ponty, M. (2006). The structure of behavior. Original work published 1942. Pittsburg: Duquesne University Press.

Moore, R. L., Baru, C., Baxter, D., Fox, G. C., Majumdar, A., Papadopoulos, P., \& Norman, M. L. (2014). Gateways to discovery: cyberinfrastructure for the long tail of science. In Proceedings of theannual conference on extreme science and engineering discovery environment (pp. 39-47). New York: ACM.

The Quantitative Methods for Psychology 
Newell, K., Deutsch, K., Sosnoff, J., \& Mayer-Kress, G. (2006). Variability in motor output as noise: a default and erroneous proposition. Movement System Variability, 99(1), 3-23.

Paxton, A. \& Dale, R. (2013). Frame-differencing methods for measuring bodily synchrony in conversation. Behavior Research Methods, 45(2), 329-343. doi:10.3758/ s13428-012-0249-2

Pincus, S. M. (1991). Approximate entropy as a measure of system complexity. Proceedings of the National Academy of Sciences, 88(6), 2297-2301. doi:10.1073/ pnas.88.6.2297

Ramseyer, F. \& Tschacher, W. (2011). Nonverbal synchrony in psychotherapy: coordinated body movement reflects relationship quality and outcome. Journal of Consulting and Clinical Psychology, 79(3), 284-295. doi:10.1037/a0023419

Ramseyer, F. \& Tschacher, W. (2014). Nonverbal synchrony of head- and body-movement in psychotherapy: different signals have different associations with outcome. Frontiers in Psychology, 5(august), 1-99.

Richman, J. S. \& Moorman, J. R. (2000). Physiological timeseries analysis using approximate entropy and sample entropy. American Journal of Physiology - Heart and Circulatory Physiology, 278(6), H2039-H2049.

Robertson, G., Caldwell, G., Hamill, J., Kamen, G., \& Whittlesey, S. (2013). Research methods in biomechanics (2nd edition). Champaign, Illinois: Human Kinetics.

Sheets-Johnstone, M. (2011). The primacy of movement. New York: John Benjamins Publishing. doi:10.1075/ aicr.82

Siegel, D. J. (2012). The developing mind: how relationships and the brain interact to shape who we are (2nd edition). New York: The Guilford Press.
Sokunbi, M. O. (2014). Sample entropy reveals high discriminative power between young and elderly adults in short fmri data sets. Frontiers in Neuroinformatics, 8, 69-89. doi:10.3389/fninf.2014.00069

Takahashi, T., Cho, R. Y., Mizuno, T., Kikuchi, M., Murata, T., Takahashi, K., \& Wada, Y. (2010). Antipsychotics reverse abnormal eeg complexity in drugnaive schizophrenia: a multiscale entropy analysis. NeuroImage, 51(1), 173-182. doi:10 . 1016 / j . neuroimage.2010.02.009

Thompson, E. (2007). Mind in life: biology, phenomenology, and the sciences of mind. Harvard: Harvard University Press.

Towns, J., Cockerill, T., Dahan, M., Foster, I., Gaither, K., Grimshaw, A., \& Peterson, G. D. (2014). Xsede: accelerating scientific discovery. Computing in Science \& Engineering, 16(5), 62-74. doi:10.1109/MCSE.2014.80

Tschacher, W., Ramseyer, F., \& Koole, S. L. (2017). Sharing the now in the social present: duration of nonverbal synchrony is linked with personality. Journal of Personality. doi:10.1111/jopy.12298

Varela, F. J., Thompson, E., \& Rosch, E. (1991). The embodied mind: cognitive science and human experience. Harvard: MIT Press.

Windolf, M., Götzen, N., \& Morlock, M. (2008). Systematic accuracy and precision analysis of video motion capturing systems-exemplified on the vicon-460 system. Journal of Biomechanics, 41(12), 2776-2780. doi:10 . 1016/j.jbiomech.2008.06.024

Winter, D. A. (2009). Biomechanics and motor control of human movement. John \& Sons: Wiley. doi:10.1002/ 9780470549148

Yu, Y. \& Lin, L. (2012). Agreement: Statistical Tools for Measuring Agreement. Retrieved from https://CRAN.Rproject.org/package=Agreement

\section{Open practices}

- The Open Material badge was earned because supplementary material(s) are available on the journal's web site.

\section{Citation}

Finn, M. T. M., Smith, C. L., \& Nash, M. R. (2018). Open-ended measurement of whole-body movement: a feasibility study. The Quantitative Methods for Psychology, 14(1), 38-54. doi:10.20982/tqmp.14.1.p038

Copyright @ 2018, Finn, Smith, and Nash. This is an open-access article distributed under the terms of the Creative Commons Attribution License (CC BY). The use, distribution or reproduction in other forums is permitted, provided the original author(s) or licensor are credited and that the original publication in this journal is cited, in accordance with accepted academic practice. No use, distribution or reproduction is permitted which does not comply with these terms.

Received: 09/10/2017 Accepted: 01/12/2017 